\title{
Kidney Transplant Biopsies for Delayed Graft Function. Does it add Therapeutic Value?
}

Vishwanath Siddagangaiah', Stavros Papachristos ${ }^{1}$, Marcus Lowe ${ }^{1}$, John Blaikley², David Van Dellen 1,2, Muir Morton', Titus Augustine ${ }^{1,2}$

1Department of Renal and Pancreatic Transplantation, Manchester Royal Infirmary \& ${ }^{2}$ University of Manchester

\section{Introduction}

- Graft biopsies are performed in kidney transplants with delayed graft function (DGF) to confirm acute tubular necrosis and exclude potential underlying rejection.

- With the increasing use of donor after cardiac death (DCD) and Extended Criteria Donors, DGF incidence is increasing whilst modern immunosuppressive regimes have reduced the incidence of acute rejection to less than $10 \%$.

- Routine biopsies may therefore not provide therapeutic efficiency or financial utility.

- They are known to be associated with potential morbidity.

\section{Methods}

- A retrospective analysis was performed of kidney transplant recipients over 4 years (2004-8).

- Patients who underwent graft biopsy due to DGF within 2 weeks of transplantation were included.

- Only biopsies performed with the aim of confirming acute tubular necrosis (ATN) and excluding rejection were considered for analysis.

\section{Discussion}

- The exclusion of rejection has traditionally mandated early biopsy following renal transplantation in the context of DGF.

- However, the incidence of concomitant rejection with DGF remains low.

- Only $12 \%$ of biopsies in our analysis revealed rejection. The indications for a routine biopsy during DGF therefore requires more critical appraisal.

- The role of novel alternate techniques, including urinary metabolomics or proteomics may provide further insights in this cohort of patients.
Results

866 Recipients
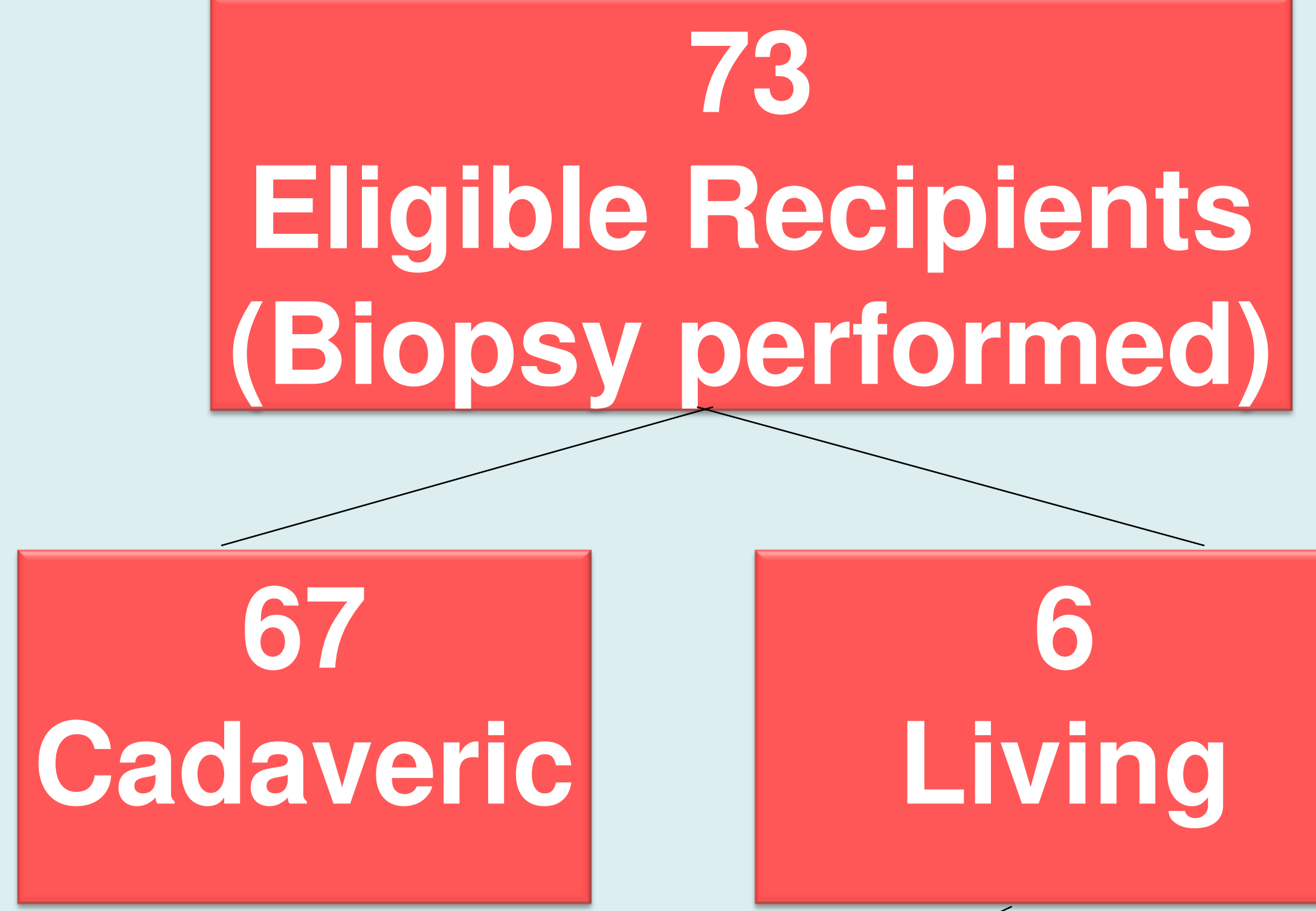

6

Living
55

(75.34\%)

ATN

73

Final Analysis

Therefore only $9 / 73(12 \%)$ of all performed biopsies demonstrated rejection.
9

Rejection

Other

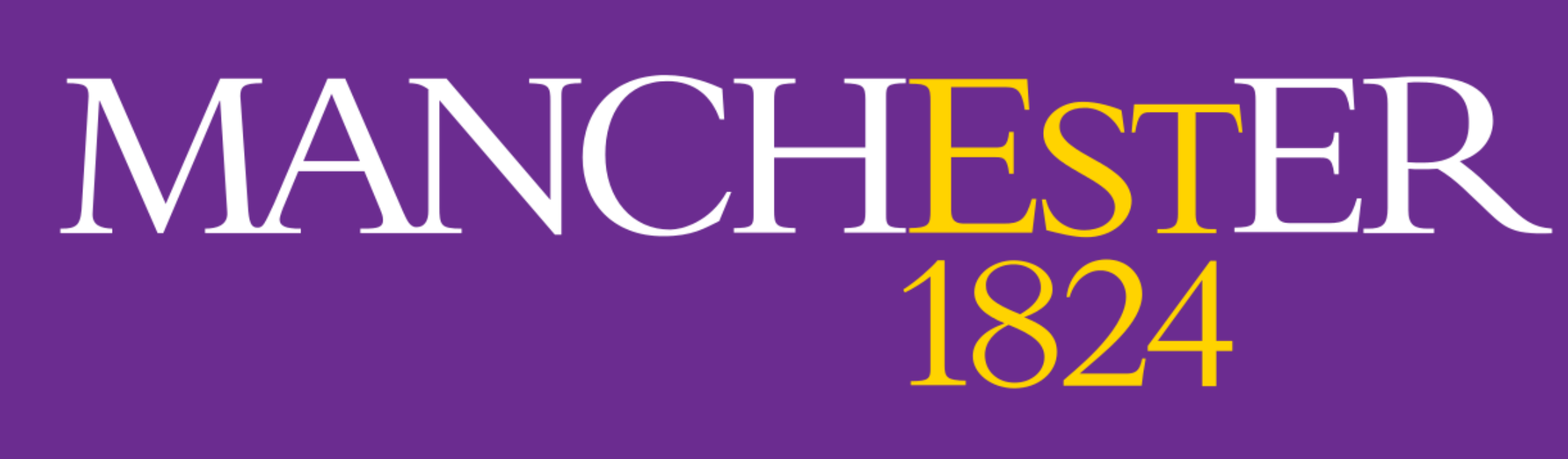

